\section{Prostate cancer screening}

To the Editor: We read with interest the article about prostate specific antigen (PSA) and screening, "Prostate cancer screening and the role of PSA: A UK perspective" by Sooriakumaran in the January 2021 issue. ${ }^{1}$ We are concerned with the author's endorsement of unproven management options, an unreferenced claim that transperineal prostate biopsies curtail antibiotic resistance, and especially the generalized take-home point that "PSA screening saves lives."

The European Randomised Study of Screening for Prostate Cancer (ERSPC), which the author used to justify screening, showed no such benefit. The relative risk for all-cause mortality was $1.00(95 \%$ confidence interval $0.98-1.02, P=.82){ }^{2}$ Only a disease-specific benefit was detected: 570 men would need to be screened to prevent 1 death of prostate cancer at 16 years in the latest follow-up of the study. ${ }^{3}$ For this marginal benefit, substantial costs are incurred. These include the psychological consequences of a cancer diagnosis, harms of prostate biopsies, and side effects of treatments including radiation, radical prostatectomy, and androgen deprivation therapy (eg, impotence or incontinence, or both). The US Preventive Services Task Force estimates that for 1,000 men ages 55 to 69 who are screened, 240 will experience the stress of an elevated PSA, 100 will be diagnosed with cancer, and at least 60 will suffer serious harm. ${ }^{4}$

Causing this harm is not inexpensive. A cascade of testing and procedures follows an elevated PSA, which by itself costs about $\$ 40$. However, additional fees can quickly add up - that of ultrasounds $(\$ 150)$, specialist consultations $(\$ 350)$, prostate biopsies $(\$ 500)$, and more. ${ }^{5}$ The United States wastes billions of dollars annually on nonbeneficial healthcare costs. We believe that higher value care is crucial for patient outcomes and for the sustainability of healthcare spending. The costs of PSA testing-both financial and to the patient's well-being-are not worth it.
Sherry Zhang, MD

University of California, San Diego

Ian Jenkins, MD

University of California, San Diego

-REFERENCES

1. Sooriakumaran P. Prostate cancer screening and the role of PSA: A UK perspective. Cleve Clin J Med 2021; 88(1)14-16. doi:10.3949/ccjm.88a.20164

2. Schröder FH, Hugosson J, Roobol MJ, et al. Screening and prostate cancer mortality: results of the European Randomised Study of Screening for Prostate Cancer (ERSPC) at 13 years of follow-up. Lancet 2014; 384(9959):2027-2035. doi:10.1016/S0140-6736(14)60525-0

3. Hugosson J, Roobol MJ, Månsson M, et al. A 16-yr Follow-up of the European Randomized study of Screening for Prostate Cancer. Eur Urol 2019; 76(1):43-51. doi:10.1016/j.eururo.2019.02.009

4. US Preventive Services Task Force; Grossman DC, Curry SJ, Owens DK, et al. Screening for prostate cancer: US Preventive Services Task Force recommendation statement. JAMA 2018; 319(18):1901-1913. doi:10.1001/ jama.2018.3710

5. American Academy of Family Physicians. The PSA blood test for prostate cancer. When men need it-and when they don't. Choosing Wisely April, 2014. Accessed March 11, 2021. https://www.choosingwisely.org/patient-resources/psa-test-for-prostate-cancer/

doi:10.3949/ccjm.88c.05001 